\begin{tabular}{|c|l|}
\hline Title & Total reaction cross section on a deuteron target and the eclipse effect of the constituent neutron and proton \\
\hline Author(s) & Horiuchi, W.; Suzuki, Y.; U esaka, T.; Miwa, M. \\
\hline Citation & $\begin{array}{l}\text { Physical Review C, 102(5), 054601 } \\
\text { https://doi.org/L0.1103/PhysRevC.102.054601 }\end{array}$ \\
\hline Issue Date & 2020-11-02 \\
\hline Doc URL & http://hdl.handle.net/2115/80012 \\
\hline Rights & @2020 A merican Physical Society \\
\hline Type & article \\
\hline File Information & PhysRevC.102-5_054601.pdf \\
\hline
\end{tabular}

Instructions for use 


\title{
Total reaction cross section on a deuteron target and the eclipse effect of the constituent neutron and proton
}

\author{
W. Horiuchi $\odot,{ }^{1, *}$ Y. Suzuki, ${ }^{2,3}$ T. Uesaka, ${ }^{3,4}$ and M. Miwa ${ }^{3,4}$ \\ ${ }^{1}$ Department of Physics, Hokkaido University, Sapporo 060-0810, Japan \\ ${ }^{2}$ Department of Physics, Niigata University, Niigata 950-2181, Japan \\ ${ }^{3}$ RIKEN Nishina Center, Wako 351-0198, Japan \\ ${ }^{4}$ Department of Physics, Saitama University, Saitama 338-8570, Japan
}

(Received 23 August 2020; accepted 19 October 2020; published 2 November 2020)

\begin{abstract}
Background: Eclipse effect of the neutron and the proton in a deuteron target is essential to correctly describe high-energy deuteron scattering. The nucleus-deuteron scattering needs information on not only the nucleusproton interaction but also the nucleus-neutron interaction, for which no direct measurement of the nucleusneutron cross sections is available for unstable nuclei.

Purpose: We systematically evaluated the total reaction cross sections on a deuteron target to explore the feasibility of extracting the nucleus-neutron interaction from measurable cross sections.

Methods: High-energy nucleus-deuteron collision is described by the Glauber model, in which the proton and neutron configuration of the deuteron is explicitly taken into account.

Results: Our calculation reproduces available experimental total reaction cross section data on the nucleusdeuteron scattering. The possibility of extracting the nucleus-neutron total reaction cross section from nucleusdeuteron and nucleus-proton total reaction cross sections is explored. The total reaction cross sections on proton, neutron, and deuteron targets can be expressed, to good accuracy, in terms of the nuclear matter radius and the neutron skin thickness. Incident-energy dependence of the total reaction cross sections is examined.

Conclusions: The total reaction cross section on a deuteron target includes information on both the nucleusneutron and the nucleus-proton profile functions. Measuring the cross sections by deuteron and proton targets is a promising tool to extract the nuclear size properties.
\end{abstract}

DOI: 10.1103/PhysRevC.102.054601

\section{INTRODUCTION}

Over half a century ago, Glauber and Franco examined high-energy antiproton-deuteron $(\bar{p} d)$ scattering and pointed out that the $\bar{p} d$ absorption cross section was always smaller than the sum of $\bar{p} p$ and $\bar{p} n$ cross sections [1,2]. This crosssection defect was significantly large and its origin was explained by the so-called "eclipse" effect that the neutron and the proton in the deuteron cast individual shadows. When either particle lies in the shadow cast by the other, it absorbs less effectively than when outside it. As stressed there, the high-energy deuteron scattering is in fact not simply explained only by the geometrical eclipse but also multiple scattering effects have to be taken into account. The total reaction cross section is a quantity mostly used to measure absorption in nuclear collisions.

The purpose of this paper is to study the total reaction cross section of a projectile nucleus $P$ scattered by a deuteron target with a beam energy of several tens of $\mathrm{MeV}$ to a few $\mathrm{GeV}$ per nucleon, especially focusing on the eclipse effect, that is, the difference of the $P d$ total reaction cross section from the sum of total reaction cross sections of $P n$ and $P p$. As the effect

\footnotetext{
*whoriuchi@nucl.sci.hokudai.ac.jp
}

is influenced by the size of $P$ and the property of underlying nucleon-nucleon interaction, e.g., the difference of the $p p$ and $p n$ total cross sections, this study is expected to be closely related to the issue of radii of neutrons and protons of $P$.

Measurements of interaction and total reaction cross sections with the help of inverse kinematics have unveiled various exotic structures of unstable nuclei, e.g., halo [3-5], neutronskin structure [6], and recently the anomalous growth of the nuclear radius of $\mathrm{Ca}$ isotopes has been found [7]. Although such measurements have mostly been done on a carbon target, a deuteron target combined with a proton target appears to be superior in extracting the radii of protons and neutrons. Furthermore, experiments on charge-changing cross sections have recently been carried out to extract information on the radius [8,9], but the experiments often pose some problems in both their data analysis and a theoretical formulation from the point of view of a reliable determination of the radius [10-13].

Thanks to the recent development of a proton target [14-16], the nucleus-proton interaction for unstable nuclei can now be studied and gives valuable input to explore the property of neutron skin thickness, the difference in radii between neutrons and protons $[17,18]$. No such study is, however, available for the nucleus-neutron interaction. By understanding quantitatively the eclipse effect of the deuteron cross section, we expect to get information on the nucleus-neutron interaction for unstable nuclei. 
The deuteron has unique advantages in that it has only one bound state, the ground state, and its wave function is readily calculated. The deuteron is fragile and can easily dissociate without inducing the mass-number change of $P$ in the collision. Such an inelastic scattering process is difficult to identify in a measurement, and thus the interaction cross section is actually measured. A theory usually calculates the total reaction cross section. We discuss the difference between both the cross sections.

The paper is organized as follows. In the next section, we present a formulation to describe the high-energy nuclear collision. Expressions for the $P d$ total reaction cross section and the interaction cross section are derived within the Glauber model, including the spatial correlations of the proton and the neutron in the deuteron. Section III shows our results. First in Sec. III A we test the validity of the present model by comparing the theoretical total reaction cross sections on a deuteron target with the available experimental data for known nuclei. We quantify the deuteron eclipse effect and investigate its magnitude systematically. In Sec. III B, we discuss the possibility of extracting the nucleus-neutron interaction from simultaneous measurement of the total reaction cross sections on deuteron and proton targets by performing a numerical "experiment". Two practical examples are given. For future measurement, we show the universal behavior of the total reaction cross sections on deuteron, proton, and neutron targets for a wide range of incident energy and mass number using realistic density distributions. Section III C relates the total reaction cross sections to the nuclear size properties, matter radius and neutron skin thickness. Sensitivity of the cross sections to the radii of protons and neutrons is quantified through an investigation of their incident-energy dependence. Section III D compares the magnitude of the total reaction and interaction cross sections. The conclusion is given in Sec. IV.

\section{GLAUBER MODEL FOR DEUTERON CROSS SECTIONS}

\section{A. Total reaction cross section}

Here we employ the Glauber model to describe the highenergy $P d$ scattering. The validity of this approach was well tested in ${ }^{58} \mathrm{Ni}-d$ scattering [19-21]. Let $\Psi_{\alpha}^{P}$ and $\Psi_{\beta}^{T}$ denote the wave functions of a projectile nucleus, $P$, and a target nucleus, $T$. The cross section from an initial state $(\alpha=0, \beta=0)$ to a final state, $\alpha$ and $\beta$, is evaluated by integrating the reaction probability over an impact parameter vector, $\boldsymbol{b}[1]$ :

$$
\sigma_{\alpha \beta}=\int d \boldsymbol{b}\left|\left\langle\Psi_{\alpha}^{P} \Psi_{\beta}^{T}\left|\prod_{j \in P} \prod_{k \in T}\left[1-\Gamma_{j k}\right]\right| \Psi_{0}^{P} \Psi_{0}^{T}\right\rangle\right|^{2} .
$$

Here, $\Gamma_{j k}=1-e^{i \chi_{N N}\left(\boldsymbol{b}+\boldsymbol{s}_{j}-\boldsymbol{s}_{k}\right)}$ is specified by a phase-shift function $\chi_{N N}$ of the nucleon-nucleon $(N N)$ scattering. $\boldsymbol{s}_{j}\left(\boldsymbol{s}_{k}\right)$ is a two-dimensional vector, perpendicular to the beam $(z)$ direction, of the nucleon coordinate relative to the center of mass of the projectile (target) nucleus. $\chi_{N N}$ depends on the $p n$ pair or the $p p$ pair. $\chi_{n n}$ is assumed to be the same as $\chi_{p p}$. The spin dependence of $\chi_{N N}$ is ignored. The parameters of $\Gamma_{j k}\left(\Gamma_{N N}\right)$ are taken from Ref. [22].

The total reaction cross section is defined by $\sigma_{T: R}=$ $\sum_{\alpha \beta} \sigma_{\alpha \beta}-\sigma_{00}$. The sum over $\alpha \beta$ is taken by using a closure relation, e.g., $\sum_{\alpha}\left|\Psi_{\alpha}^{P}\right\rangle\left\langle\Psi_{\alpha}^{P}\right|=1$, and the unitarity of the $N N$ phase-shift function, $\left|e^{i \chi_{N N}}\right|^{2}=1$, leading to

$$
\sigma_{T: R}=\int d \boldsymbol{b}\left[1-P_{P T}(\boldsymbol{b})\right]
$$

where $P_{P T}(\boldsymbol{b})$ is the probability for the elastic scattering

$$
P_{P T}(\boldsymbol{b})=\left|\left\langle\Psi_{0}^{P} \Psi_{0}^{T}\left|\prod_{j \in P} \prod_{k \in T}\left[1-\Gamma_{j k}\right]\right| \Psi_{0}^{P} \Psi_{0}^{T}\right\rangle\right|^{2} .
$$

Although the evaluation of $P_{P T}(\boldsymbol{b})$ is in general hard, a nucleus- $N$ case can be done to good accuracy. Given the proton and neutron densities of the projectile nucleus $P$, we get the $P N$ phase-shift function $\chi_{N}^{P}$ by $[1,21]$

$$
\begin{aligned}
i \chi_{N}^{P}(\boldsymbol{b}) & =\ln \left\langle\Psi_{0}^{P}\left|\prod_{j \in P}\left[1-\Gamma_{j N}\right]\right| \Psi_{0}^{P}\right\rangle \\
& \approx-\int d \boldsymbol{r}\left[\rho_{p}^{P}(\boldsymbol{r}) \Gamma_{p N}(\boldsymbol{b}+\boldsymbol{s})+\rho_{n}^{P}(\boldsymbol{r}) \Gamma_{n N}(\boldsymbol{b}+\boldsymbol{s})\right],
\end{aligned}
$$

where $\boldsymbol{r}=(\boldsymbol{s}, z)$. This approximation works well when the fluctuation of $\chi_{N}^{P}$ is small enough [19-21,23]. In fact, the ansatz (4) that relates $\chi_{N}^{P}$ to the proton and neutron densities of $P$ has proven to work well for many cases of $P N$ scattering [24-27]. The $P N$ total reaction cross section $\sigma_{N}(N=n, p)$ reads

$$
\sigma_{N}=\int d \boldsymbol{b}\left[1-P_{N}(\boldsymbol{b})\right]
$$

with

$$
P_{N}(\boldsymbol{b})=\left|e^{i \chi_{N}^{P}(\boldsymbol{b})}\right|^{2} .
$$

A unique advantage of the deuteron target is that we can calculate Eq. (3) accurately using its ground-state wave function $\phi_{d}(\boldsymbol{r})$. The $P d$ total reaction cross section turns out to be

$$
\sigma_{d}=\int d \boldsymbol{b}\left[1-P_{d}(\boldsymbol{b})\right],
$$

where

$$
P_{d}(\boldsymbol{b})=\left.\left.\left|\int d \boldsymbol{r}\right| \phi_{d}(\boldsymbol{r})\right|^{2} e^{i \chi_{p}^{P}\left(\boldsymbol{b}+\frac{1}{2} s\right)+i \chi_{n}^{P}\left(\boldsymbol{b}-\frac{1}{2} s\right)}\right|^{2} .
$$

We use the AV8' potential [28] to generate $\phi_{d}(\boldsymbol{r}) .\left|\phi_{d}(\boldsymbol{r})\right|^{2}$ is actually defined by taking an average with respect to relevant magnetic quantum numbers and by integrating out spin-isospin coordinates. In this way we get a quantitative evaluation of the cross-section defect:

$$
\delta \sigma=\sigma_{p}+\sigma_{n}-\sigma_{d} .
$$

\section{B. Interaction cross section}

Experimentally observed in most cases is the interaction cross section but not the total reaction cross section. Because it is hard to calculate the interaction cross section at the same accuracy as the total reaction cross section, it is of practical importance to set a theoretical limit on the interaction cross section. The interaction cross section is a semi-inclusive cross 
section defined by summing over all $\alpha$ 's but particle-bound states (b.s.):

$$
\sigma_{T: I}=\sum_{\alpha \beta} \sigma_{\alpha \beta}-\sum_{\alpha \in \text { b.s. } \beta} \sigma_{\alpha \beta}=\sigma_{T: R}-\Delta \sigma,
$$

where

$$
\Delta \sigma=\sum_{\alpha \in \text { b.s. } \beta} \sigma_{\alpha \beta}-\sigma_{00} .
$$

A calculation of $\Delta \sigma$ demands all b.s. wave functions of the projectile nucleus, which is in general hopeless. Provided the projectile nucleus has only one b.s., its ground state $\sigma_{T: I}$ is equal to $\sigma_{T: R}-\Delta_{0} \sigma$, where

$$
\Delta_{0} \sigma=\sum_{\beta} \sigma_{0 \beta}-\sigma_{00}
$$

In other cases $\Delta_{0} \sigma$ is the lower bound of $\Delta \sigma: \Delta_{0} \sigma \leqq \Delta \sigma$. Even the calculation of $\Delta_{0} \sigma$ is difficult in a general case. For the deuteron target, however, with the same ingredients as needed to calculate $\sigma_{d}$, we can get it. To show this, we again use the closure relation for all target states and the approximation of Eq. (4) to the phase-shift function:

$$
\begin{aligned}
\sum_{\beta} \sigma_{0 \beta} & =\int d \boldsymbol{b}\left\langle\left.\Psi_{0}^{T}||\left\langle\Psi_{0}^{P}\left|\prod_{j \in P} \prod_{k \in T}\left[1-\Gamma_{j k}\right]\right| \Psi_{0}^{P}\right\rangle\right|^{2} \mid \Psi_{0}^{T}\right\rangle \\
& \simeq \int d \boldsymbol{b} P_{0}(\boldsymbol{b}),
\end{aligned}
$$

where

$$
P_{0}(\boldsymbol{b})=\int d \boldsymbol{r}\left|\phi_{d}(\boldsymbol{r})\right|^{2}\left|e^{i \chi_{p}^{P}\left(\boldsymbol{b}+\frac{1}{2} s\right)+i \chi_{n}^{P}\left(\boldsymbol{b}-\frac{1}{2} s\right)}\right|^{2} .
$$

Substitution of this result into Eq. (12) leads to

$$
\Delta_{0} \sigma=\int d \boldsymbol{b}\left[P_{0}(\boldsymbol{b})-P_{d}(\boldsymbol{b})\right] .
$$

\section{RESULTS AND DISCUSSION}

\section{A. Test of $\sigma_{d}$ calculation}

First, we compare in Table I the $\sigma_{d}$ values with experiment for some well-known nuclei: ${ }^{12} \mathrm{C},{ }^{16} \mathrm{O}$, and ${ }^{40} \mathrm{Ca}$. The values in parentheses denote the results with the Coulomb breakup cross sections. The center-of-masscorrected harmonic-oscillator type densities are employed for those nuclei [25], and the oscillator parameters needed to

TABLE I. Total reaction cross sections of ${ }^{12} \mathrm{C},{ }^{16} \mathrm{O}$, and ${ }^{40} \mathrm{Ca}$ on a deuteron target at $50 \mathrm{MeV} /$ nucleon. The experimental cross sections at $48.7 \pm 0.15 \mathrm{MeV} /$ nucleon are taken from Ref. [29]. The cross section in parentheses includes the Coulomb breakup contribution of the deuteron that is estimated following Ref. [18].

\begin{tabular}{lcrr}
\hline \hline & Glauber & OLA & Expt. [29] \\
\hline${ }^{12} \mathrm{C}$ & $638(642)$ & 687 & $600 \pm 17$ \\
${ }^{16} \mathrm{O}$ & $735(741)$ & 795 & $726 \pm 21$ \\
${ }^{40} \mathrm{Ca}$ & $1220(1260)$ & 1310 & $1260 \pm 30$ \\
\hline \hline
\end{tabular}

specify the densities are set to reproduce empirical charge radii [30]. Although the data are at low incident energies, the Glauber-model calculation reproduces them very well. The inclusion of the Coulomb breakup contribution leads to results closer to experiment especially for ${ }^{40} \mathrm{Ca}$. Our $\sigma_{d}$ value for ${ }^{40} \mathrm{Ca}$ is in excellent agreement with $1270 \mathrm{mb}$, which is the cross section obtained by a continuum-discretized-coupledchannels calculation [31] that includes the Coulomb breakup.

Table I also lists the cross sections calculated with an optical-limit approximation (OLA) [1,21], which uses

$$
P_{d}^{\mathrm{OLA}}(\boldsymbol{b})=\left|e^{i \int d \boldsymbol{r}\left[\rho_{p}^{d}(\boldsymbol{r}) \chi_{p}^{P}(\boldsymbol{b}+s)+\rho_{n}^{d}(\boldsymbol{r}) \chi_{n}^{P}(\boldsymbol{b}+s)\right]}\right|^{2},
$$

instead of Eq. (8), where $\rho_{N}^{d}$ is the nucleon density of the deuteron. Because the OLA takes only the first term in the cumulant expansion, this approximation is not expected to work well in the $P d$ scattering, where the deuteron wave function is spatially extended $[19,20,23]$. Apparently the OLA tends to overestimate $\sigma_{d}$ significantly.

In what follows we neglect the Coulomb breakup contribution, although its inclusion is possible in the Glauber model as discussed in Refs. [18,32-35]. It should be noted that the most important Coulomb effect for heavy projectiles is the deuteron breakup: It increases $\sigma_{d}$ by a few percent for the projectile nucleus with its proton number $Z_{P} \approx 20$ and by about $5 \%$ for $Z_{P} \approx 40$ [18].

Figure 1 displays $\sigma_{d}, \sigma_{p}, \sigma_{n}$, and $\delta \sigma$ for (a) ${ }^{16} \mathrm{O}$, (b) ${ }^{40} \mathrm{Ca}$, and (c) ${ }^{30} \mathrm{Ne}$ as a function of incident energy. Note that $\sigma_{n}$ is equal to $\sigma_{p}$ for ${ }^{16} \mathrm{O}$ and ${ }^{40} \mathrm{Ca}$ because the neutron and proton densities are identical. The density distribution of ${ }^{30} \mathrm{Ne}$, taken from Refs. [17,36], is obtained by the Hartree-Fock (HF) calculation with the $\mathrm{SkM}^{*}$ effective interaction [37]. Note that the density of ${ }^{30} \mathrm{Ne}$ reproduces reasonably well the observed cross section on a carbon target at $240 \mathrm{MeV} /$ nucleon [38]. The $\sigma_{p}$ value of ${ }^{30} \mathrm{Ne}$ is close to that of ${ }^{40} \mathrm{Ca}$, which is due to the fact that $\sigma_{p}$ is most sensitive to the neutron density of the projectile nucleus and both ${ }^{30} \mathrm{Ne}$ and ${ }^{40} \mathrm{Ca}$ have almost the same matter radius. In contrast to $\sigma_{p}, \sigma_{n}$ for ${ }^{30} \mathrm{Ne}$ is smaller than that for ${ }^{40} \mathrm{Ca}$ as understood from different proton numbers of those projectile nuclei.

For the above three projectile nuclei, $\sigma_{d}$ is always significantly smaller than $\sigma_{p}+\sigma_{n}$ : The $\delta \sigma$ value is about $10-30 \%$ of $\sigma_{p}+\sigma_{n}$. The larger the mass number of the projectile nucleus is, the larger the $\delta \sigma$ value that is obtained, which is naturally understood from the eclipse effect: The chance that the proton and the neutron in the deuteron cast individual shadows increases with increasing mass number of the projectile nucleus. The energy dependence of $\delta \sigma$ follows that of the $N N$ total cross section $\sigma_{N N}^{\text {tot }}$ that specifies $\Gamma_{N N}$ (see, e.g., Ref. [39]). $\delta \sigma$ reaches a minimum at $\approx 200-500 \mathrm{MeV} /$ nucleon, where $\sigma_{N N}^{\text {tot }}$ also becomes a minimum.

A simple estimate of $\delta \sigma$ for the $\bar{p} d$ case was given by $\delta \sigma=$ $2 \sigma_{p} \sigma_{n}\left\langle\frac{1}{4 \pi r^{2}}\right\rangle_{d}[1,2]$, where $\left\langle\frac{1}{r^{2}}\right\rangle_{d}$ stands for the expectation value of the inverse square of the neutron-proton distance $r$ by the deuteron ground state. With our present value, $\left\langle\frac{1}{r^{2}}\right\rangle_{d}=$ $0.294 \mathrm{fm}^{-2}=0.0294 \mathrm{mb}^{-1}$, we find that the formula significantly overestimates the cross-section defect, about 5 times larger than the present one. The formula was actually derived under the assumption that the size of the deuteron is larger 


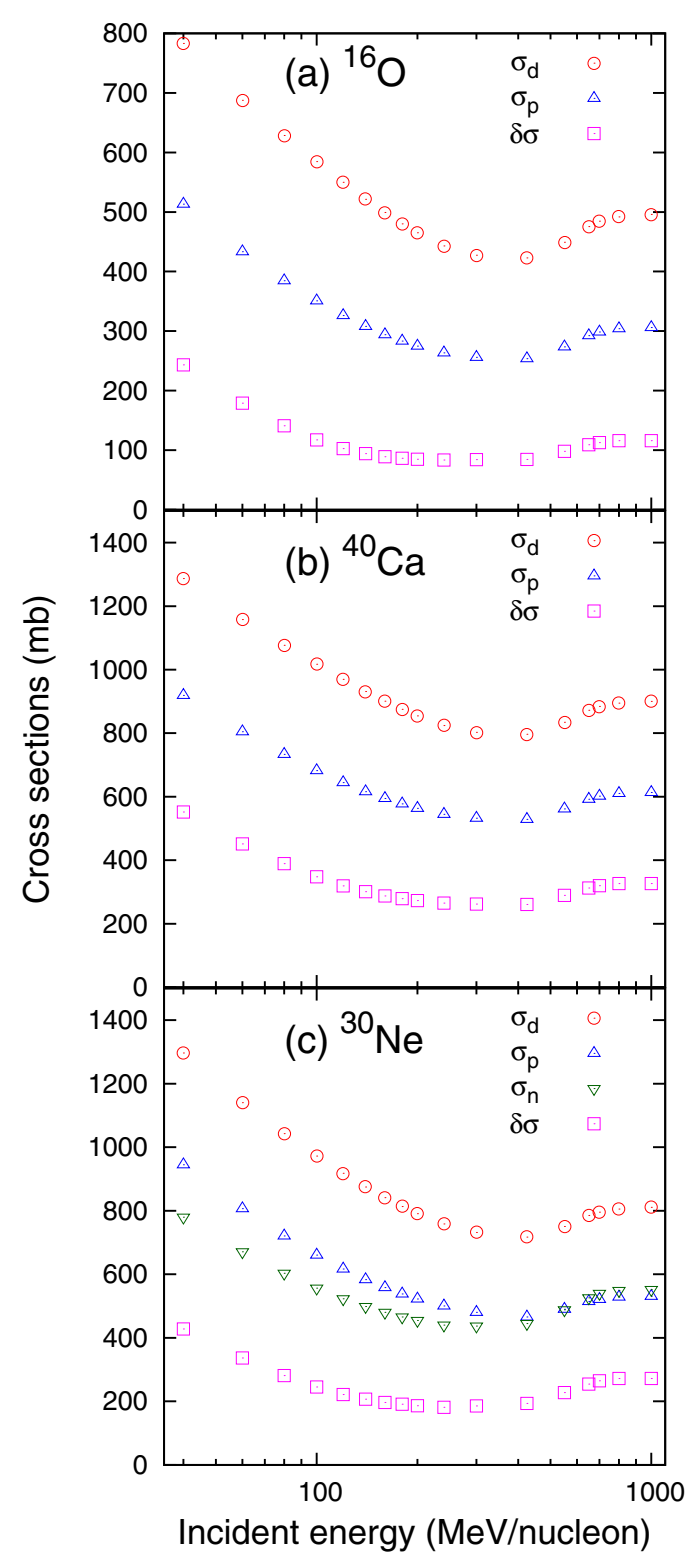

FIG. 1. Total reaction cross sections, $\sigma_{d}, \sigma_{p}$, and $\sigma_{n}$ of the projectile nuclei of (a) ${ }^{16} \mathrm{O}$, (b) ${ }^{40} \mathrm{Ca}$, and (c) ${ }^{30} \mathrm{Ne}$ as a function of incident energy. $\sigma_{n}$ is equal to $\sigma_{p}$ for ${ }^{16} \mathrm{O}$ and ${ }^{40} \mathrm{Ca}$. The cross-section defect $\delta \sigma$ is also plotted.

than the range of the $\bar{p} N$ interaction. The assumption is not valid in our case because the range of the $P N$ interaction is at most comparable or larger than the size of the deuteron.

\section{B. Numerical experiment: Extracting $\sigma_{n}$}

Suppose that both $\sigma_{p}$ and $\sigma_{d}$ are measured experimentally. An interesting question is whether or not we can extract $\sigma_{n}$ from those observed cross sections. To discuss its possibility, we perform a numerical experiment by taking examples of the $N_{P}=2 Z_{P}$ projectile nucleus, ${ }^{30} \mathrm{Ne}$ and ${ }^{60} \mathrm{Ca}$, where the difference between $\sigma_{p}$ and $\sigma_{n}$ is expected to be large especially at low incident energy because of the large neutron skin thickness expected.

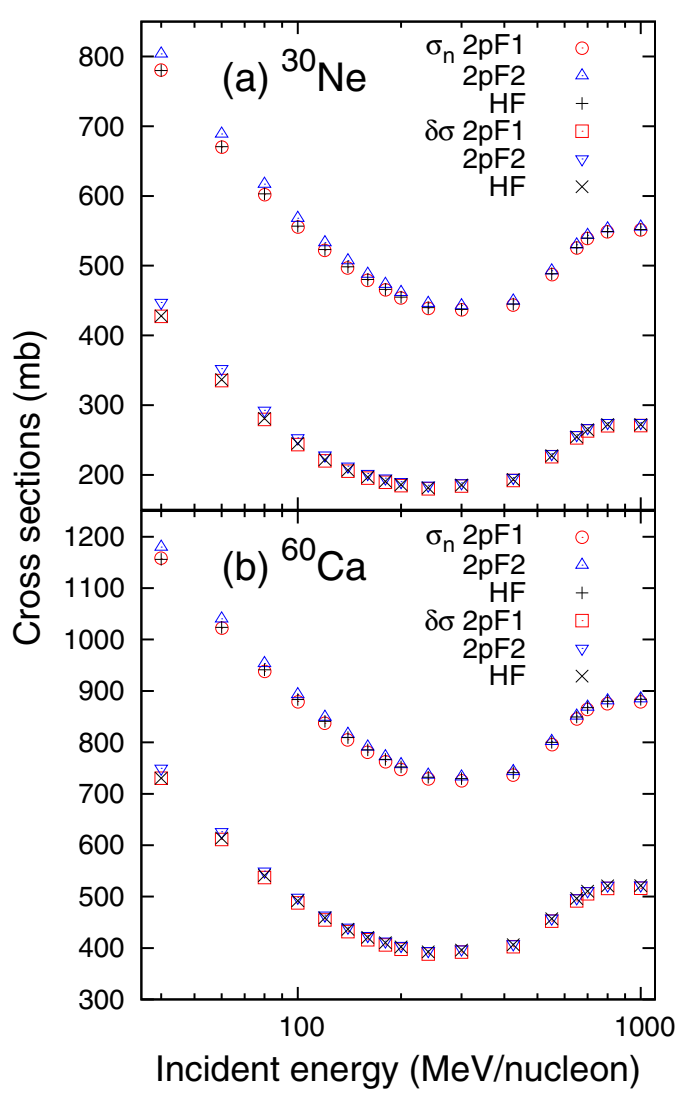

FIG. 2. Comparison between "theory" $(2 \mathrm{pF} 1$ and $2 \mathrm{pF} 2)$ and "experiment" (HF) for the total reaction cross section $\sigma_{n}$ of (a) ${ }^{30} \mathrm{Ne}$ and (b) ${ }^{60} \mathrm{Ca}$ and the cross-section defect $\delta \sigma .2 \mathrm{pF} 1(2 \mathrm{pF} 2)$ stands for the cross sections determined so as to reproduce $\sigma_{p}$ and $\sigma_{d}$ values at selected incident energies, assuming different (conditional) Fermi-type density distributions for neutrons and protons. See text for detail.

We assume $\sigma_{p}$ and $\sigma_{d}$ calculated with the Hartree-Fock (HF) density distribution to be experimental data, denoted as $\sigma_{p}^{\mathrm{HF}}$ and $\sigma_{d}^{\mathrm{HF}}$, respectively. We attempt to determine $\sigma_{n}$ under the assumption that no knowledge on the density distribution or even the radius of $P$ is given. In any case we need the density of $P$, and as a reasonable choice we assume a two-parameter Fermi $(2 \mathrm{pF})$ function for neutrons and protons $(N=n, p)$ :

$$
\rho_{N}(r)=\frac{\rho_{0 N}}{1+\exp \left[\left(r-R_{N}\right) / d_{N}\right]},
$$

where $R_{N}$ and $d_{N}$ are radius and diffuseness parameters, and the central density $\rho_{0 N}$ is determined by the condition, e.g., for proton $4 \pi \int_{0}^{\infty} d r r^{2} \rho_{p}(r)=Z_{P}$. We consider two cases below.

\section{Case I: Data available at two incident energies}

We may assume different parameters for the Fermi functions of neutrons and protons, and we determine them so as to reproduce the "observed" $\sigma_{d}^{\mathrm{HF}}$ and $\sigma_{p}^{\mathrm{HF}}$ values at two incident energies, chosen at 100 and $200 \mathrm{MeV} /$ nucleon. Whether this procedure is successful or not is judged by comparing the 
resulting "theoretical" values of $\sigma_{n}$ and $\delta \sigma$ with $\sigma_{n}^{\mathrm{HF}}$ and $\delta \sigma^{\mathrm{HF}}$. Plots labeled $2 \mathrm{pF} 1$ in Fig. 2 denote the theoretical values for the incident energy of 40 to $1000 \mathrm{MeV} /$ nucleon, whereas plots labeled HF denote observed ones. Both cases of (a) ${ }^{30} \mathrm{Ne}$ and (b) ${ }^{60} \mathrm{Ca}$ projectile nuclei show excellent agreement: The $2 \mathrm{pF}$ density distributions reproduce the HF cross sections of ${ }^{30} \mathrm{Ne}$ and ${ }^{60} \mathrm{Ca}$ for all the incident energies despite the fact that the four parameters of the Fermi functions are determined to reproduce the experimental data only at two incident energies. This numerical experiment strongly corroborates that the evaluation of $\sigma_{d}$ is reliable enough to predict $\sigma_{n}$ correctly even at different incident energies.

The extracted parameters $\left(R_{n}, d_{n}\right)$ and $\left(R_{p}, d_{p}\right)$ are, in fm, $(3.20,0.66)$ and $(3.08,0.50)$ for ${ }^{30} \mathrm{Ne}$ and $(4.33,0.65)$ and $(4.03,0.52)$ for ${ }^{60} \mathrm{Ca}$, respectively. We see different surface diffuseness for neutrons and protons and large neutron skin thickness for both nuclei. Let us define the matter radius $r_{m}$ and the neutron skin thickness $\delta r$ of $P$ in terms of the rootmean-square radii of neutrons and protons, $r_{n}\left(N_{P}, Z_{P}\right)$ and $r_{p}\left(N_{P}, Z_{P}\right)$, by

$$
\begin{aligned}
& r_{m}=\sqrt{\frac{Z_{P}}{A_{P}} r_{p}^{2}\left(N_{P}, Z_{P}\right)+\frac{N_{P}}{A_{P}} r_{n}^{2}\left(N_{P}, Z_{P}\right),} \\
& \delta r=r_{n}\left(N_{P}, Z_{P}\right)-r_{p}\left(N_{P}, Z_{P}\right),
\end{aligned}
$$

where $A_{P}=N_{P}+Z_{P}$. The resulting values are $\left(r_{m}, \delta r\right)=$ $(3.34,0.47)$ for ${ }^{30} \mathrm{Ne}$ and $(3.98,0.46)$ for ${ }^{60} \mathrm{Ca}$, in excellent agreement with the $\mathrm{HF}$ values $(3.34,0.47)$ and $(4.00,0.46)$, respectively.

For the present analysis to be sensitive enough to the choice of the parameters of the Fermi function, one of the incident energies should be chosen $\lesssim 300 \mathrm{MeV} /$ nucleon, because $\sigma_{p n}^{\text {tot }}$ is then much larger than $\sigma_{p p}^{\text {tot }}$ and reduces possible uncertainty in determining the parameters $[17,18]$.

\section{Case II: Data available at only one incident energy}

If we have experimental data, $\sigma_{p}$ and $\sigma_{d}$, at only one incident energy, say $100 \mathrm{MeV} /$ nucleon, we put on a constraint, e.g., $d_{n}=d_{p}=0.6 \mathrm{fm}$, and attempt to reproduce the experimental data using two free parameters, $R_{n}$ and $R_{p}$. The extracted radius parameters $\left(R_{n}, R_{p}\right)$ are, in fm, $(3.42,2.90)$ for ${ }^{30} \mathrm{Ne}$ and $(4.49,3.88)$ for ${ }^{60} \mathrm{Ca}$, respectively. Though the matter radii $r_{m}$ of these isotopes are 3.37 and 4.01 in fair agreement with the $\mathrm{HF}$ values, the resulting $\delta r$ values turn out to be 0.30 for ${ }^{30} \mathrm{Ne}$ and 0.39 for ${ }^{60} \mathrm{Ca}$, which are considerably smaller than the HF values. As shown in Fig. 2, the calculated $\sigma_{n}$ value denoted $2 \mathrm{pF} 2$ still reproduces the $\mathrm{HF}$ value reasonably well at higher incident energies but deviates from $\sigma_{n}^{\mathrm{HF}}$ with decreasing incident energies, e.g., by about $2 \%$ at $40 \mathrm{MeV} /$ nucleon. The quality of the theoretical prediction of

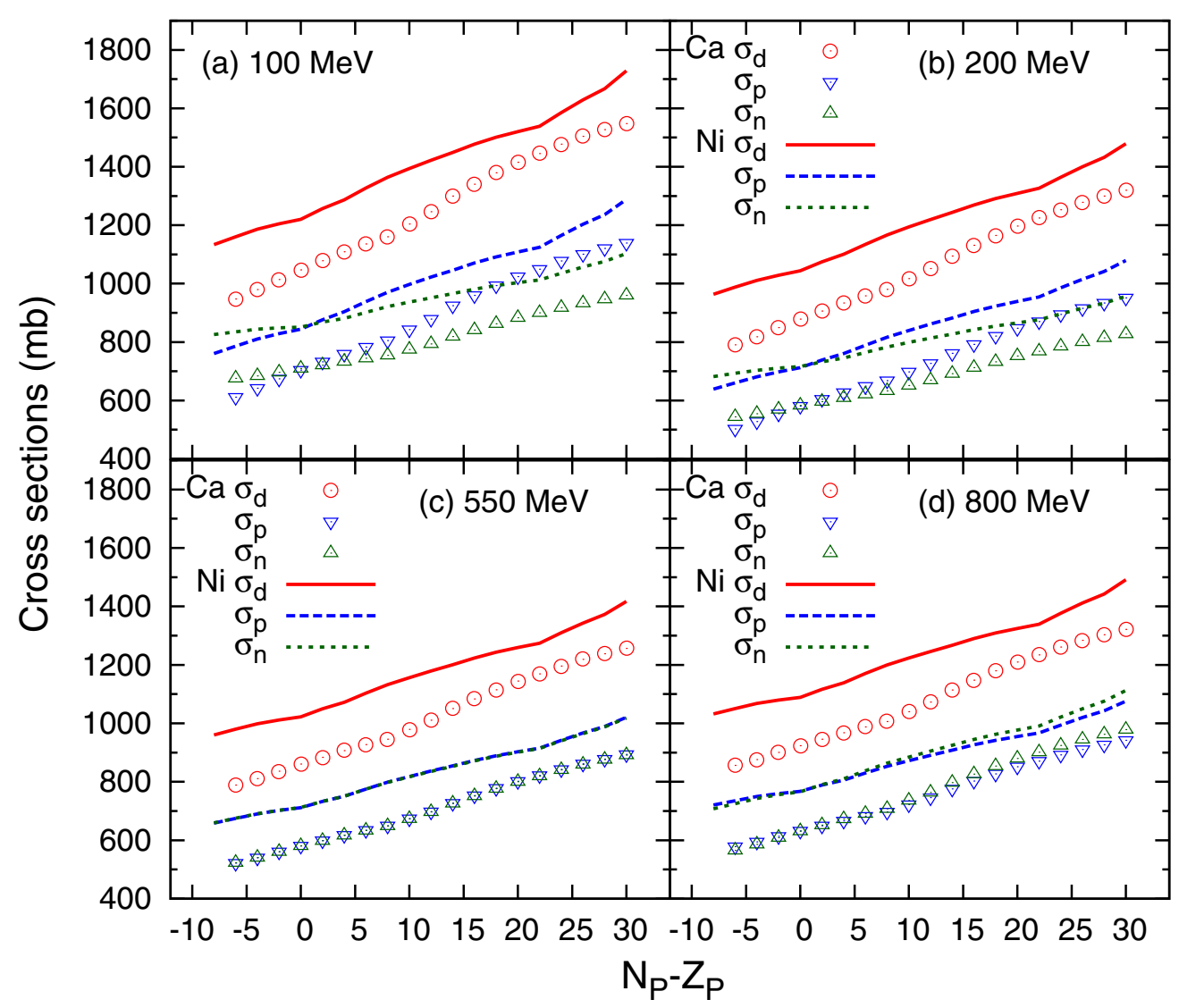

FIG. 3. Total reaction cross sections of $\sigma_{d}, \sigma_{p}$, and $\sigma_{n}$ for Ca and Ni isotopes at the incident energies of (a) 100, (b) 200, (c) 550, and (d) 800 $\mathrm{MeV} /$ nucleon as a function of neutron excess $N_{P}-Z_{P}$. Note that it is hard to see the difference between $\sigma_{p}$ and $\sigma_{n}$ at $550 \mathrm{MeV} / \mathrm{nucleon}$. 
course depends on the incident energy chosen for the data, and it should be as low as $\lesssim 300 \mathrm{MeV} /$ nucleon.

As confirmed in the Case I study, we have succeeded in calculating consistently $\sigma_{d}, \sigma_{p}$, and $\sigma_{n}$ cross sections at all incident energies. In Fig. 3 we exhibit those cross sections for ${ }^{34-70} \mathrm{Ca}$ and ${ }^{48-86} \mathrm{Ni}$ as a function of neutron excess $N_{P}-Z_{P}$. The densities of $\mathrm{Ca}$ and $\mathrm{Ni}$ isotopes are based on the HF calculation [17,36]. In so far as the HF calculation gives $r_{m}$ and $\delta r$ close to realistic values, the cross sections in the figure are expected to show general features of real cross sections. For example, the cross-section ratio $\sigma_{p} / \sigma_{n}$ shows an interesting variation against the incident energy $E$ : The ratio is larger than 1 for $N_{P}-Z_{P}>0$ and smaller than 1 for $N_{P}-Z_{P}<0$ at both $E=100$ and $200 \mathrm{MeV} /$ nucleon, whereas it is almost 1 at $E=550 \mathrm{MeV} /$ nucleon and gets smaller than 1 at 800 $\mathrm{MeV} /$ nucleon. This variation of the ratio exactly follows that of $\sigma_{p n}^{\text {tot }} / \sigma_{p p}^{\text {tot }}$.

We note, however, that the uncertainties of the present $\sigma_{d}$ data are approximately $2-3 \%$ [29], which are the same order or even larger than the deviation obtained in the Case II study. Improvement of the experimental accuracy is highly desired for precise determination of $\sigma_{n}$ as it was already achieved $\lesssim 1 \%$ for the interaction cross-section measurements involving unstable nuclei by proton [16] and carbon [7] targets.

\section{Relating $\sigma_{T: R}$ to $r_{m}$ and $\delta r$}

The numerical analysis of Case I has confirmed that $\sigma_{n}$ or even $\left(r_{m}, \delta r\right)$ values of a projectile nucleus $P$ can be reproduced quite well once $\sigma_{d}$ and $\sigma_{p}$ of $P$ are given. This strongly suggests that the total reaction cross section $\sigma_{T: R}$ of $P$ can be determined by $r_{m}$ and $\delta r$ to good accuracy: Introducing a reaction radius $a_{T}$ by $\sigma_{T: R}=\pi a_{T}^{2}$, we may conjecture that $a_{T}$ is expressed as

$$
a_{T}=\alpha_{T}(E) r_{m}+\beta_{T}(E) \delta r+\gamma_{T}(E) .
$$

Here, $\alpha_{T}(E), \beta_{T}(E)$, and $\gamma_{T}(E)$ depend on the incident energy $E$ but do not depend on the projectile nucleus. The conjecture has actually been confirmed successfully in Refs. $[17,18]$ for proton and ${ }^{12} \mathrm{C}$ targets. We extend that analysis to determine the coefficients for the deuteron and neutron targets by covering the projectile nuclei ${ }^{14-24} \mathrm{O},{ }^{18-34} \mathrm{Ne},{ }^{20-40} \mathrm{Mg},{ }^{22-46} \mathrm{Si}$, ${ }^{26-50} \mathrm{~S},{ }^{34-70} \mathrm{Ca}$, and ${ }^{48-86} \mathrm{Ni}$. See Refs. $[17,18]$ for details.

Figure 4 compares those coefficients for deuteron, proton, and neutron targets. $\alpha_{T}$ depends on $E$ weakly, which is understood by noting that $\sigma_{T: R}$ is roughly equal to $\pi r_{m}^{2}$. The coefficient $\beta_{T}$ among others is most important to probe $\delta r$. The larger $\beta_{T}$ is in its magnitude, the more accurately $\delta r$ is determined. The $E$ dependence of $\beta_{T}$ follows that of $\sigma_{N N}^{\text {tot }}$ : The ratio $\sigma_{p n}^{\text {tot }} / \sigma_{p p}^{\text {tot }}$ is about 2.5 at $100 \mathrm{MeV} /$ nucleon, 2 at $200 \mathrm{MeV} /$ nucleon, about 1 at $\approx 550 \mathrm{MeV} /$ nucleon, and less than 1 beyond $800 \mathrm{MeV} /$ nucleon. The apparent symmetry of $\beta_{n}$ and $\beta_{p}$ is understood by noting that $\sigma_{n}$ can also be obtained from $\chi_{p}^{P}(\boldsymbol{b})$ of Eq. (4) with $\rho_{p}^{P}(\boldsymbol{r})$ and $\rho_{n}^{P}(\boldsymbol{r})$ being exchanged.

What is noteworthy in Fig. 4 is that $\beta_{d}$ has fairly large values especially at lower energies, which is different from the ${ }^{12} \mathrm{C}$ target [17]. The larger sensitivity of the deuteron target is probably because the deuteron is spatially extended and may probe sensitively the surface region of the projectile

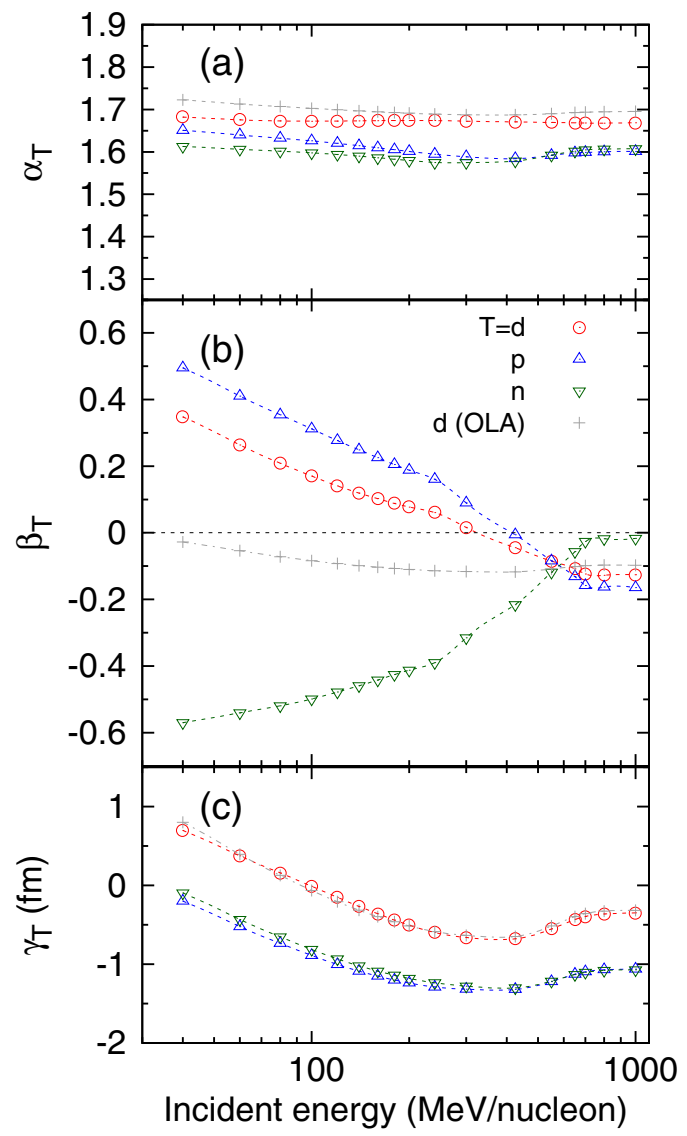

FIG. 4. Incident-energy dependence of coefficients (a) $\alpha_{T}$, (b) $\beta_{T}$, and (c) $\gamma_{T}$ of the reaction radius $a_{T}$ of Eq. (20), where $T$ denotes a target nucleus. d(OLA) stands for the coefficients obtained when $\sigma_{d}$ is calculated by the OLA.

nucleus. If $\sigma_{d}$ is, however, calculated by OLA, Eq. (17), the resulting coefficients differ from those discussed above. The coefficients given in the OLA calculation are also displayed in the figure. We find that $\beta_{d}$ with OLA is found to be small and almost constant, $\approx-0.1$, beyond $100 \mathrm{MeV} /$ nucleon. It is better to avoid OLA calculations for an analysis with $\sigma_{d}$ data.

\section{Difference between $\sigma_{d}$ and $\sigma_{d: I}$}

As already mentioned, the deuteron target has the advantage that the upper bound of the interaction cross section can be evaluated reliably using the deuteron wave function, that is, $\sigma_{d: I} \leqq \sigma_{d}-\Delta_{0} \sigma$, where $\Delta_{0} \sigma$ is calculated by using Eq. (16). Figure 5 displays $\sigma_{d}, \sigma_{d}-\Delta_{0} \sigma$ (the upper bound of $\sigma_{d: I}$ ), and $\Delta_{0} \sigma$ for (a) ${ }^{16} \mathrm{O}$, (b) ${ }^{40} \mathrm{Ca}$, and (c) ${ }^{30} \mathrm{Ne}$ projectile nuclei as a function of incident energy. $\Delta_{0} \sigma$ has a maximum at $80 \mathrm{MeV} /$ nucleon and its magnitude is $60-70 \mathrm{mb}$, which is about $10 \%$ of $\sigma_{d}$ for ${ }^{16} \mathrm{O}, 7 \%$ for ${ }^{40} \mathrm{Ca}$, and $6 \%$ for ${ }^{30} \mathrm{Ne}$, respectively. $\Delta_{0} \sigma$ decreases with increasing incident energy and has a minimum at $425 \mathrm{MeV} /$ nucleon. It looks like the ratio $\Delta_{0} \sigma / \sigma_{d}$ has a minimum at $550 \mathrm{MeV} /$ nucleon. Beyond $300 \mathrm{MeV} /$ nucleon, the ratio is at most a few percent for both ${ }^{40} \mathrm{Ca}$ and ${ }^{30} \mathrm{Ne}$. 


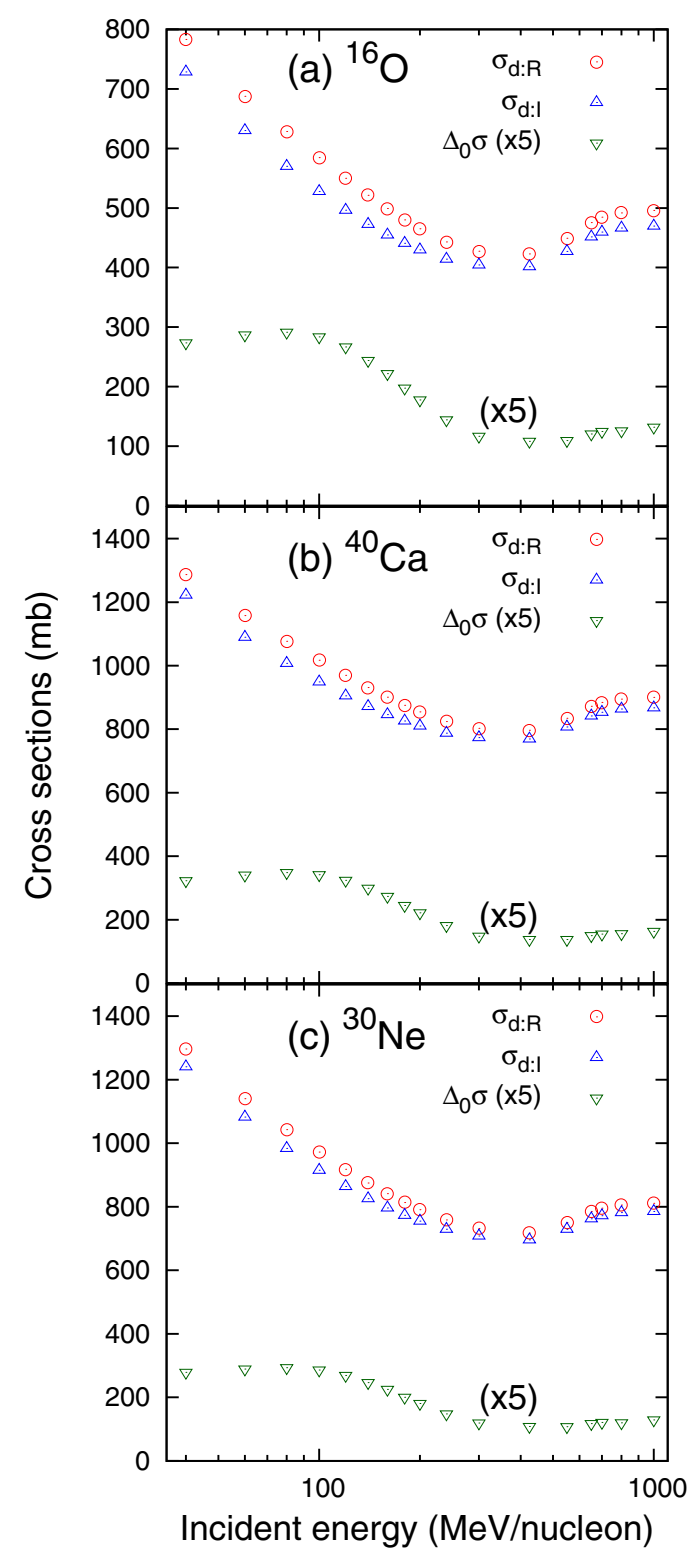

FIG. 5. Total reaction cross section $\sigma_{d}$ and interaction cross section $\sigma_{d: I}$ for (a) ${ }^{16} \mathrm{O}$, (b) ${ }^{40} \mathrm{Ca}$, and (c) ${ }^{30} \mathrm{Ne}$ projectile nuclei as a function of incident energy. $\sigma_{d: I}$ shown here is actually $\sigma_{d}-\Delta_{0} \sigma$, which is equal to the upper bound of $\sigma_{d: I} . \Delta_{0} \sigma$ multiplied by 5 is also plotted.

\section{CONCLUSION}

We have systematically investigated the total reaction cross sections scattered by a deuteron target using the Glauber model. The eclipse effect cast by the neutron and the proton in the deuteron is explicitly taken into account. The interaction cross section is also evaluated in the same framework.

The calculated total reaction cross sections reasonably well reproduce the available experimental data. By extending the analysis to cover many nuclei, $\mathrm{O}$ to $\mathrm{Ni}$, we find that the nucleus-deuteron cross section is considerably smaller than the sum of cross sections of nucleus-neutron and nucleusproton. The cross-section defect is understood by the eclipse effect.

The cross-section defect carries information on the nucleus-proton and nucleus-neutron profile functions. Because of this, the nucleus-neutron cross section can be extracted by simultaneous measurements of the total reaction cross sections by both deuteron and proton targets. We have convincingly shown that measuring both cross sections at two incident energies is the best choice to determine the neutron cross section or the nuclear size properties. Energy dependence of the total reaction cross section is given in terms of the matter radius and the neutron skin thickness of the projectile nucleus.

Measuring the total reaction cross sections by both deuteron and proton targets is the most unambiguous way to determine the neutron and proton radii of unstable nuclei. We note that for the unstable nuclei near the drip line that have only one bound state, the theoretical interaction cross section can be obtained at good accuracy. In the present analysis, we ignore the Coulomb breakup contribution of the deuteron target, which will be significant for heavy projectiles. The inclusion of this effect in the Glauber model is straightforward and will be reported elsewhere.

\section{ACKNOWLEDGMENTS}

We thank K. Ogata for valuable communications. This work was in part supported by JSPS KAKENHI Grants No. 18K03635, No. 18H04569, and No. 19H05140. W.H. acknowledges the Collaborative Research Program 2020, Information Initiative Center, Hokkaido University.
[1] R. J. Glauber, Lectures in Theoretical Physics, edited by W. E. Brittin and L. G. Dunham (Interscience, New York, 1959), Vol. 1, p. 315.

[2] V. Franco and R. J. Glauber, Phys. Rev. 142, 1195 (1966).

[3] I. Tanihata, H. Hamagaki, O. Hashimoto, Y. Shida, N. Yoshikawa, K. Sugimoto, O. Yamakawa, T. Kobayashi, and N. Takahashi, Phys. Rev. Lett. 55, 2676 (1985).

[4] I. Tanihata, H. Savajols, and R. Kanungo, Prog. Part. Nucl. Phys. 68, 215 (2013), and references therein.

[5] S. Bagchi, R. Kanungo, Y. K. Tanaka, H. Geissel, P. Doornenbal, W. Horiuchi, G. Hagen, T. Suzuki, N. Tsunoda, D. S. Ahn et al., Phys. Rev. Lett. 124, 222504 (2020).
[6] T. Suzuki, H. Geissel, O. Bochkarev, L. Chulkov, M. Golovkov, D. Hirata, H. Irnich, Z. Janas, H. Keller, T. Kobayashi et al., Phys. Rev. Lett. 75, 3241 (1995).

[7] M. Tanaka, M. Takechi, M. Fukuda, D. Nishimura, T. Suzuki, Y. Tanaka, T. Moriguchi, D. S. Ahn, A. Aimaganbetov, M. Amano et al., Phys. Rev. Lett. 124, 102501 (2020).

[8] A. Estradé, R. Kanungo, W. Horiuchi, F. Ameil, J. Atkinson, Y. Ayyad, D. Cortina-Gil, I. Dillmann, A. Evdokimov, F. Farinon et al., Phys. Rev. Lett. 113, 132501 (2014).

[9] R. Kanungo, W. Horiuchi, G. Hagen, G. R. Jansen, P. Navrátil, F. Ameil, J. Atkinson, Y. Ayyad, D. Cortina-Gil, I. Dillmann et al., Phys. Rev. Lett. 117, 102501 (2016). 
[10] A. Bhagwat and Y. K. Gambhir, Phys. Rev. C 69, 014315 (2004).

[11] T. Yamaguchi, I. Hachiuma, A. Kitagawa, K. Namihira, S. Sato, T. Suzuki, I. Tanihata, and M. Fukuda, Phys. Rev. Lett. 107, 032502 (2011).

[12] S. Terashima, I. Tanihata, R. Kanungo, A. Estradé, W. Horiuchi, F. Ameil, J. Atkinson, Y. Ayyad, D. Cortina-Gil, I. Dillmann et al., Prog. Theor. Exp. Phys. 2014, 101D02 (2014).

[13] Y. Suzuki, W. Horiuchi, S. Terashima, R. Kanungo, F. Ameil, J. Atkinson, Y. Ayyad, D. Cortina-Gil, I. Dillmann, A. Estradé et al., Phys. Rev. C 94, 011602(R) (2016).

[14] D. Nishimura, M. Fukuda, M. Takechi, D. Mihara, J. Ishikawa, K. Komurasaki, R. Matsuta, T. Matsumiya, M. Kubokic, T. Yoshitake et al., Nucl. Phys. A 834, 470c (2010).

[15] T. Moriguchi, A. Ozawa, S. Ishimoto, Y. Abe, M. Fukuda, I. Hachiuma, Y. Ishibashi, Y. Ito, T. Kuboki, M. Lantz et al., Phys. Rev. C 88, 024610 (2013).

[16] T. Moriguchi, M. Amano, A. Ozawa, W. Horiuchi, Y. Abe, T. Fujii, R. Kagesawa, D. Kamioka, A. Kitagawa, M. Mukai et al., Nucl. Phys. A 994, 121663 (2020).

[17] W. Horiuchi, Y. Suzuki, and T. Inakura, Phys. Rev. C 89, 011601(R) (2014).

[18] W. Horiuchi, S. Hatakeyama, S. Ebata, and Y. Suzuki, Phys. Rev. C 93, 044611 (2016).

[19] K. Yabana, Y. Ogawa, and Y. Suzuki, Nucl. Phys. A 539, 295 (1992).

[20] K. Yabana, Y. Ogawa, and Y. Suzuki, Phys. Rev. C 45, 2909 (1992).

[21] Y. Suzuki, R. G. Lovas, K. Yabana, and K. Varga, Structure and Reactions of Light Exotic Nuclei (Taylor \& Francis, London, 2003).

[22] B. Abu-Ibrahim, W. Horiuchi, A. Kohama, and Y. Suzuki, Phys. Rev. C 77, 034607 (2008); 80, 029903(E) (2009); 81, 019901(E) (2010).

[23] Y. Ogawa, K. Yabana, and Y. Suzuki, Nucl. Phys. A 543, 722 (1992).
[24] K. Varga, S. C. Pieper, Y. Suzuki, and R. B. Wiringa, Phys. Rev. C 66, 034611 (2002).

[25] B. Abu-Ibrahim, S. Iwasaki, W. Horiuchi, A. Kohama, and Y. Suzuki, J. Phys. Soc. Jpn. 78, 044201 (2009).

[26] T. Nagahisa and W. Horiuchi, Phys. Rev. C 97, 054614 (2018)

[27] S. Hatakeyama and W. Horiuchi, Nucl. Phys. A 985, 20 (2019).

[28] R. B. Wiringa, V. G. J. Stoks, and R. Schiavilla, Phys. Rev. C 51, 38 (1995).

[29] A. Auce, R. F. Carlson, A. J. Cox, A. Ingemarsson, R. Johansson, P. U. Renberg, O. Sundberg, and G. Tibell, Phys. Rev. C 53, 2919 (1996).

[30] I. Angeli and K. P. Marinova, At. Data Nucl. Tables 99, 69 (2013).

[31] K. Minomo, K. Washiyama, and K. Ogata, J. Nucl. Sci. Technol. 54, 127 (2019).

[32] J. Margueron, A. Bonaccorso, and D. M. Brink, Nucl. Phys. A 720, 337 (2003).

[33] B. Abu-Ibrahim and Y. Suzuki, Prog. Theor. Phys. 112, 1013 (2004); 114, 901 (2005).

[34] P. Capel, D. Baye, and Y. Suzuki, Phys. Rev. C 78, 054602 (2008).

[35] W. Horiuchi, S. Hatakeyama, S. Ebata, and Y. Suzuki, Phys. Rev. C 96, 024605 (2017).

[36] W. Horiuchi, T. Inakura, T. Nakatsukasa, and Y. Suzuki, Phys. Rev. C 86, 024614 (2012).

[37] J. Bartel, P. Quentin, M. Brack, C. Guet, and H. Håkansson, Nucl. Phys. A 386, 79 (1982).

[38] M. Takechi, T. Ohtsubo, T. Kuboki, M. Fukuda, D. Nishimura, T. Suzuki, T. Yamaguchi, A. Ozawa, T. Moriguchi, T. Sumikawa et al., Mod. Phys. Lett. A 25, 1878 (2010).

[39] M. Tanabashi, K. Hagiwara, K. Hikasa, K. Nakamura, Y. Sumino, F. Takahashi, J. Tanaka, K. Agashe, G. Aielli, C. Amsler et al. (Particle Data Group), Phys. Rev. D 98, 030001 (2018), and 2019 update. 